\title{
CALCULATION OF TORSIONAL AND ROTATIONAL RAMAN SPECTRA OF HYDROGEN PEROXIDE
}

\author{
G. A. Pitsevich, ${ }^{a^{*}}$ M. B. Shundalau, ${ }^{a}$ and D. S. Umreiko ${ }^{b}$
}

UDC 539.192:530.145.7

We present calculated intensity distributions in torsional, rotational, and torsional-rotational Raman lines in spectra of hydrogen peroxide. Ab initio calculations of polarizability tensor components as functions of internal rotation angle were carried out in the HF/6-311G approximation. It is shown that the structure and transformational properties of the polarizability tensor components of hydrogen peroxide in extended molecular symmetry group $G_{4}(E M)$ permit formation of purely rotational and torsional and rotational-torsional Raman spectra. Common expressions to calculate Raman line intensities governed by torsional and rotational motions of the non-rigid symmetric top molecule are obtained. The torsional components of the line intensities have been calculated by estimating the appropriate matrix elements. The contribution of rotational components has been calculated using the 3j-symbols technique.

Keywords: hydrogen peroxide, Raman spectrum, polarizability tensor, rotational spectrum, torsional-rotational spectrum, 3j-symbols, ab initio calculations.

Introduction. The analysis, calculation, and interpretation of torsional-vibrational IR spectra of hydrogen peroxide, the simplest member of the class of non-rigid molecules, have been the subject of numerous experimental and theoretical studies [1-5]. Similar studies using Raman spectroscopy (including experimental spectra recorded in the gas phase with the required resolution) have not been reported. Recent research has shown [6] that the frequencies and intensities of the $\mathrm{H}_{2} \mathrm{O}_{2}$ torsional-rotational IR spectrum can be successfully calculated because the dependences of the molecular dipole moment components in a fixed molecular coordinate system on the internal rotation angle are known. A similar approach can be used to model the torsional-rotational Raman spectrum of $\mathrm{H}_{2} \mathrm{O}_{2}$. However, in this instance the dependences of the polarizability tensor components on the torsional angle must be found. The appearance of such capability in recent versions of the quantum-chemical GAMESS packet [7, 8] enabled the frequencies and intensities of Raman lines due to rotational, torsional, and torsional-rotational motion of the $\mathrm{H}_{2} \mathrm{O}_{2}$ molecule to be calculated.

Method. The Raman line intensity for the transition $i^{\prime \prime} \rightarrow i^{\prime}$ is known to be defined as [9]:

$$
I\left(i^{\prime \prime}, i^{\prime}\right)=\frac{2^{8} \pi^{5}\left[v \pm v\left(i^{\prime \prime}, i^{\prime}\right)\right]^{4}}{3 c^{4}} I_{0} N^{\prime \prime} S\left(i^{\prime \prime}, i^{\prime}\right),
$$

where $I_{0}$ is the intensity of the exciting radiation; $N^{\prime \prime}$, the population of the initial torsional-rotational energy level; $S\left(i^{\prime \prime}, i^{\prime}\right)$, the line strength associated with this torsional-rotational transition; $v$, the frequency of the exciting radiation; and $v\left(i^{\prime \prime}, i^{\prime}\right)$, the frequency of the Raman line. The symbol $i$ denotes the whole set of torsional $n(n=0,1,2, \ldots), \tau$ $(\tau=1,2,3,4)$, and rotational quantum numbers $J, k$, and $m$; where even values of $k$ correspond to $\tau=1$ and 4 ; uneven, $\tau=2$ and 3 [4]. The symmetric top model was used to describe the rotational motion because the $\mathrm{H}_{2} \mathrm{O}_{2}$ molecule is a slightly asymmetric top (degree of asymmetry $\kappa=-0.99223$ ) $[1,4]$.

*To whom correspondence should be addressed.

${ }^{\mathrm{a} B e l a r u s i a n}$ State University, 4 Nezavisimosti Ave., Minsk, 220030, Belarus; e-mail: pitsevich@bsu.by; shundalov@bsu.by; ${ }^{\mathrm{b}}$ A. N. Sevchenko Institute of Applied Physical Problems, Belarusian State University, Minsk, Belarus; e-mail: lab_dozator@mail.ru. Translated from Zhurnal Prikladnoi Spektroskopii, Vol. 77, No. 1, pp. 53-62, January- February, 2010. Original article submitted September 4, 2009. 
The line strength $S\left(i^{\prime \prime}, i^{\prime}\right)$ in the polarizability approximation is determined by the matrix element of component $\alpha_{j l}(j, l=x, y, z)$ of the static electrical polarizability tensor $\alpha_{L}$ in the spatially fixed coordinate system (CS) $L$ :

$$
S\left(i^{\prime \prime}, i^{\prime}\right)=\left|\left\langle\Psi_{e v t r}^{\prime \prime}\left|\alpha_{L}\right| \Psi_{e v t r}^{\prime}\right\rangle\right|^{2}=\left|\left\langle\Psi_{e}^{\prime \prime} \Psi_{v}^{\prime \prime} \Psi_{t}^{\prime \prime} \Psi_{r}^{\prime \prime}\left|\alpha_{L}\right| \Psi_{e}^{\prime} \Psi_{v}^{\prime} \Psi_{t}^{\prime} \Psi_{r}^{\prime}\right\rangle\right|^{2}
$$

where $\Psi_{\text {evtr }}^{\prime \prime}$ and $\Psi_{\text {evtr }}^{\prime}$ are electronic-vibrational-torsional-rotational wave functions of states $i^{\prime \prime}$ and $i^{\prime}$.

Because the electronic and vibrational states do not change during torsional-rotational transitions, the integration in Eq. (2) can be carried out over electronic and vibrational coordinates and the designation $\left\langle\Psi_{e}^{\prime \prime} \Psi_{v}^{\prime \prime}\left|\alpha_{L}\right| \Psi_{e}^{\prime} \Psi_{v}^{\prime}\right\rangle$ $=\alpha_{0 L}$ can be introduced. Then, Eq. (2) becomes:

$$
S\left(i^{\prime \prime}, i^{\prime}\right)=\left|\left\langle\Psi_{t}^{\prime \prime} \Psi_{r}^{\prime \prime}\left|\alpha_{0 L}\right| \Psi_{t}^{\prime} \Psi_{r}^{\prime}\right\rangle\right|^{2}
$$

It is more convenient for further calculations to write components of tensor $\alpha_{j l}$ in an irreducible spherical tensor form. The static electrical polarizability tensor is symmetric and has a non-zero trace. Therefore, only six of nine irreducible spherical tensor operators remain: $\alpha_{s}^{(0,0)}$ and $\alpha_{s}^{(2, \sigma)}(\sigma=0, \pm 1, \pm 2)$. These are expressed as components of the polarizability tensor in a cartesian CS as:

$$
\begin{gathered}
\alpha_{s}^{(0,0)}=\frac{1}{\sqrt{3}}\left(\alpha_{s x x}+\alpha_{s y y}+\alpha_{s z z}\right), \\
\alpha_{s}^{(2,0)}=\frac{1}{\sqrt{6}}\left(2 \alpha_{s z z}-\alpha_{s x x}-\alpha_{s y y}\right), \\
\alpha_{s}^{(2, \pm 1)}=\frac{1}{2}\left(\left(\alpha_{s x z}+\alpha_{s z x}\right) \pm i\left(\alpha_{s y z}+\alpha_{s z y}\right)\right), \\
\alpha_{s}^{(2, \pm 2)}=\frac{1}{2}\left(\left(\alpha_{s x x}-\alpha_{s y y}\right) \pm i\left(\alpha_{s x y}+\alpha_{s y x}\right)\right) .
\end{gathered}
$$

Expressions (4)-(7) are valid for both a spatially fixed CS $(s=L)$ and a fixed molecular CS $(s=m)$. The quantities $\alpha_{s}^{(0,0)}$ and $\alpha_{s}^{(2, \sigma)}$ are transformed according to representations $D^{(0)}$ and $D^{(2)}$ in a three-dimensional rotation group. However, because products $x x, y y$, and $z z$ are transformed according to a fully symmetric representation in an expanded molecular symmetry (EMS) group $G_{4}(\mathrm{EM})[10], \alpha_{s}^{(0,0)}$ and $\alpha_{s}^{(2, \sigma)}$ are also transformed according to the fully symmetric representation $A_{g s}$ of this group.

Because the $\mathrm{H}_{2} \mathrm{O}_{2}$ molecule has a second-order symmetry axis for any torsion angle $\gamma$, the Neyman theorem can be used to show that the only off-diagonal element of the polarizability tensor that is different from zero in a fixed molecular CS will be $\alpha_{m x z}$. Therefore, Eqs. (4) and (5) are not changed in CS $m$ whereas Eqs. (6) and (7) become

$$
\begin{gathered}
\alpha_{m}^{(2, \pm 1)}=\alpha_{m x z}, \\
\alpha_{m}^{(2, \pm 2)}=\frac{1}{2}\left(\alpha_{m x x}-\alpha_{m y y}\right) .
\end{gathered}
$$

Obviously in this instance $\alpha_{m}^{(2, \pm 2)}$ is also transformed according to fully symmetric representation $A_{g s}$ in group $G_{4}(E M)$. Componentwill be transformed according to $A_{g d}$ in this group because the product $x z$ is also transformed according to this representation $\alpha_{m}^{(2, \pm 1)}$.

Thus, if the polarizability tensor components are written in irreducible spherical tensor form, then Eq. (3) is expanded over the sum of the two terms: 


$$
\begin{gathered}
S\left(i^{\prime \prime}, i^{\prime}\right)=S\left(n^{\prime \prime}, \tau^{\prime \prime}, J^{\prime \prime}, k^{\prime \prime}, m^{\prime \prime} ; n^{\prime}, \tau^{\prime}, J^{\prime}, k^{\prime}, m^{\prime}\right) \\
=\sum_{m^{\prime}, m^{\prime \prime}}\left|\left\langle n^{\prime \prime}, \tau^{\prime \prime}, J^{\prime \prime}, k^{\prime \prime}\left|\alpha_{0 L}^{(0,0)}\right| n^{\prime}, \tau^{\prime}, J^{\prime}, k^{\prime}\right\rangle\right|^{2}+\sum_{\sigma=-2}^{2} \sum_{m^{\prime}, m^{\prime \prime}}\left|\left\langle n^{\prime \prime}, \tau^{\prime \prime}, J^{\prime \prime}, k^{\prime \prime}\left|\alpha_{0 L}^{(2, \sigma)}\right| n^{\prime}, \tau^{\prime}, J^{\prime}, k^{\prime}\right\rangle\right|^{2} .
\end{gathered}
$$

The variables in Eq. (10) can be gathered by converting from spatially fixed CS $L$ to fixed molecular CS $m$. Then, polarizability tensor components in irreducible spherical tensor form are transformed as follows:

$$
\alpha_{0 L}^{(q, \sigma)}=\sum_{\sigma^{\prime}=-q}^{q} \tilde{D}_{\sigma \sigma^{\prime}}^{(q)} \alpha_{0 m}^{\left(q, \sigma^{\prime}\right)}
$$

where $D_{\sigma \sigma^{\prime}}^{(q)}$ is a matrix of rotations of rank $q$.

Because the quantity $\alpha_{0 L}^{(0,0)}$ is a scalar, it is invariant to a transformation of the CS. Then, Eq. (10) becomes:

$$
S\left(i^{\prime \prime}, i^{\prime}\right)=\left\langle n^{\prime \prime}, \tau^{\prime \prime}\left|\alpha_{0 m}^{(0,0)}\right| n^{\prime}, \tau^{\prime}\right\rangle^{2} \sum_{m^{\prime}, m^{\prime \prime}}\left|\left\langle J^{\prime \prime}, k^{\prime \prime} \mid J^{\prime}, k^{\prime}\right\rangle\right|^{2}+\sum_{\sigma=-2}^{2} \sum_{m^{\prime}, m^{\prime \prime}}\left|\left\langle n^{\prime \prime}, \tau^{\prime \prime}, J^{\prime \prime}, k^{\prime \prime}\left|\sum_{\sigma^{\prime}=-2}^{2} \tilde{D}_{\sigma \sigma^{\prime}}^{(2)} \alpha_{0 m}^{\left(2, \sigma^{\prime}\right)}\right| n^{\prime}, \tau^{\prime}, J^{\prime}, k^{\prime}\right\rangle\right|^{2} \text {. }
$$

The first term in Eq. (11) is responsible for the strength of lines related to the isotropic Raman (torsional wave functions are assumed to be real); the second, anisotropic. Thus, the isotropic Raman is related to element $\alpha_{0 m}^{(0,0)}$ that is transformed according to $A_{g s}$. Therefore, the condition $\Delta \tau=0$ must be satisfied so that the integral over the torsional functions will be different from zero. The selection rules in the rotational spectrum are known [11] to be determined by the values of $q$ and $\sigma$ in the definition of irreducible spherical tensor operator $\alpha_{0 m}^{(q, \sigma)}$ :

$$
\Delta J=-q, \quad-(q+1), \ldots, q-1, q ; \Delta k=\sigma .
$$

Because $q=0$ and $\sigma=0$ for the isotropic Raman, it is obvious that the rotational state does not change during the corresponding transition. However, the torsional state can change because $\Delta n$ adopts any values. Thus, the examined polarizability tensor element produces the purely torsional spectrum of $\mathrm{H}_{2} \mathrm{O}_{2}$. Levels with the same symmetry type are combined. In this instance, the expression for the line strength becomes:

$$
\begin{gathered}
S\left(n^{\prime \prime}, \tau^{\prime \prime}, J^{\prime \prime}, k^{\prime \prime}, m^{\prime \prime} ; n^{\prime}, \tau^{\prime \prime} J^{\prime \prime}, k^{\prime \prime}, m^{\prime}\right)=\left\langle n^{\prime \prime}, \tau^{\prime \prime}\left|\alpha_{0 m}^{(0,0)}\right| n^{\prime}, \tau^{\prime \prime}\right\rangle^{2} \sum_{m^{\prime}, m^{\prime \prime}}\left|\left\langle J^{\prime \prime}, k^{\prime \prime} \mid J^{\prime \prime}, k^{\prime \prime}\right\rangle\right|^{2} \\
=\left\langle n^{\prime \prime}, \tau^{\prime \prime}\left|\alpha_{0 m}^{(0,0)}\right| n^{\prime}, \tau^{\prime \prime}\right\rangle^{2}\left(2 J^{\prime \prime}+1\right)^{2}\left(\begin{array}{ccc}
J^{\prime \prime} & 0 & J^{\prime \prime} \\
k^{\prime \prime} & 0 & -k^{\prime \prime}
\end{array}\right)^{2} .
\end{gathered}
$$

One of the $\sigma^{\prime}$ values in the second term of Eq. (11), which determines the anisotropic scattering, should be fixed according to the literature [11] (for example, let $\sigma^{\prime}=p$ ). Then, the summation over $\sigma^{\prime}$ disappears. By performing the summation over $\sigma, m^{\prime}$, and $m^{\prime \prime}$, we obtain a general expression for the line strength for the transition $n^{\prime \prime}, \tau^{\prime \prime}$, $J^{\prime \prime}, k^{\prime \prime} \rightarrow n^{\prime}, \tau^{\prime}, J^{\prime}, k^{\prime \prime}+p:$

$$
S\left(n^{\prime \prime}, \tau^{\prime \prime}, J^{\prime \prime}, k^{\prime \prime} ; n^{\prime}, \tau^{\prime} J^{\prime}, k^{\prime \prime}+p\right)=\left(2 J^{\prime \prime}+1\right)\left(2 J^{\prime}+1\right)\left\langle n^{\prime \prime}, \tau^{\prime \prime}\left|\alpha_{0 m}^{(2, p)}\right| n^{\prime}, \tau^{\prime}\right\rangle^{2}\left(\begin{array}{ccc}
J^{\prime \prime} & 2 & J^{\prime} \\
k^{\prime \prime} & p & -\left(k^{\prime \prime}+p\right)
\end{array}\right)^{2}
$$


Thus, the line-strength calculation consists of the calculation of $3 j$-symbols that arise during calculation of the integrals over rotational wave functions in addition to integrals over torsional states from the polarizability tensor components. However, the final result depends considerably on the molecular symmetry and the nature of the interaction of torsional and rotational motions in the actual molecule. It has been shown [4] that a change of torsional state is accompanied by a change of rotational and vice versa, despite the fact that the general expression for the line strength in the IR absorption spectrum of $\mathrm{H}_{2} \mathrm{O}_{2}$ does not prohibit the existence of the purely rotational and purely torsional spectra.

For the intensity of the line related to transition $n^{\prime \prime}, \tau^{\prime \prime}, J^{\prime \prime}, k^{\prime \prime} \rightarrow n^{\prime}, \tau^{\prime \prime}, J^{\prime \prime}, k^{\prime \prime}$ (isotropic Raman), Equation (1) becomes:

$$
\begin{aligned}
I=G N_{n^{\prime \prime}} \tau^{\prime \prime} & \left\langle n^{\prime \prime}, \tau^{\prime \prime}\left|\alpha_{0 m}^{(0,0)}\right| n^{\prime}, \tau^{\prime \prime}\right\rangle^{2} \sum_{J^{\prime \prime}, k^{\prime \prime}} N_{J^{\prime \prime} k^{\prime \prime}}\left(2 J^{\prime \prime}+1\right)^{2}\left(\begin{array}{ccc}
J^{\prime \prime} & 0 & J^{\prime \prime} \\
k^{\prime \prime} & 0 & -k^{\prime \prime}
\end{array}\right)^{2} \\
= & G N_{n^{\prime \prime} \tau^{\prime \prime}}\left\langle n^{\prime \prime}, \tau^{\prime \prime}\left|\alpha_{0 m}^{(0,0)}\right| n^{\prime}, \tau^{\prime \prime}\right\rangle^{2} \sum_{J^{\prime \prime}, k^{\prime \prime}} N_{J^{\prime \prime} k^{\prime \prime}}\left(2 J^{\prime \prime}+1\right),
\end{aligned}
$$

where it is considered that the square of the corresponding $3 j$-symbol is $\left(2 J^{\prime \prime}+1\right)^{-1}$ and the following designation is introduced

$$
G=\frac{2^{8} \pi^{5}\left[v \pm v\left(i^{\prime \prime}, i^{\prime}\right)\right]^{4}}{3 c^{4}} I_{0} .
$$

Considering that even values of $k$ correspond to $\tau=1$ and 4 ; uneven, $\tau=2$ and 3 , some of the terms in Eq. (12) disappear. The summation over $J$ and $k$ in Eq. (12) should formally be carried out to infinity. However, the contributions of terms with large $J$ and $k$ values begin to decrease because the population of the corresponding rotational states also decreases quickly. Therefore, the summation is performed until the total sum does not vary noticeably.

The anisotropic Raman is produced by three types of irreducible spherical tensor operator elements, $\alpha_{0 m}^{(2,0)}$, $\alpha_{0 m}^{(2, \pm 1)}$, and $\alpha_{0 m}^{(2, \pm 2)}$. Let us examine element $\alpha_{0 m}^{(2,0)}$. Because it is transformed according to $A_{g s}, \Delta \tau=0$. Quantum number $k$ also does not change whereas $\Delta J=-2,-1,0,1,2$. If quantum number $J$ does not change $(\Delta J=0)$, then the purely torsional spectrum is observed, like for isotropic scattering. The differences will consist of the values of the torsional integrals and the $3 j$-symbols. In this instance, the line intensities are:

$$
I=G N_{n^{\prime \prime} \tau^{\prime \prime}}\left\langle n^{\prime \prime}, \tau^{\prime \prime}\left|\alpha_{0 m}^{(2,0)}\right| n^{\prime}, \tau^{\prime \prime}\right\rangle^{2} \sum_{J^{\prime \prime}, k^{\prime \prime}} N_{J^{\prime \prime} k^{\prime \prime}}\left(2 J^{\prime \prime}+1\right)^{2}\left(\begin{array}{ccc}
J^{\prime \prime} & 0 & J^{\prime \prime} \\
k^{\prime \prime} & 0 & -k^{\prime \prime}
\end{array}\right)^{2} .
$$

If the rotational state changes $(\Delta J= \pm 1, \pm 2)$ and the torsional state does not, then the purely rotational spectrum of a symmetric-top molecules will form. The general formula for the line intensities in this instance is

$$
I=G \sum_{n^{\prime \prime}, \tau^{\prime \prime}} N_{n^{\prime \prime} \tau^{\prime \prime}}\left\langle n^{\prime \prime}, \tau^{\prime \prime}\left|\alpha_{0 m}^{(2,0)}\right| n^{\prime \prime}, \tau^{\prime \prime}\right\rangle^{2} \sum_{k^{\prime \prime}} N_{J^{\prime \prime} k^{\prime \prime}}\left(2 J^{\prime \prime}+1\right)\left(2 J^{\prime}+1\right)\left(\begin{array}{ccc}
J^{\prime \prime} & 0 & J^{\prime} \\
k^{\prime \prime} & 0 & -k^{\prime \prime}
\end{array}\right)^{2} .
$$

However, in the general case both the torsional and rotational states change. The intensity will be summed for various allowed $k$ values because the value of quantum number $k$ does not change. Then, the general formula for the line intensity of the torsional-rotational spectrum is:

$$
I=G N_{n^{\prime \prime} \tau^{\prime \prime}}\left\langle n^{\prime \prime}, \tau^{\prime \prime}\left|\alpha_{0 m}^{(2,0)}\right| n^{\prime}, \tau^{\prime \prime}\right\rangle^{2} \sum_{k^{\prime \prime}} N_{J^{\prime \prime} k^{\prime \prime}}\left(2 J^{\prime \prime}+1\right)\left(2 J^{\prime}+1\right)\left(\begin{array}{ccc}
J^{\prime \prime} & 0 & J^{\prime} \\
k^{\prime \prime} & 0 & -k^{\prime \prime}
\end{array}\right)^{2} .
$$


Let us examine element $\alpha_{0 m}^{(2, \pm 1)}$. As noted above, it is transformed according to $A_{g d}$. Because of this, the torsional state must change. Then, values $\tau=1 \leftrightarrow \tau=2$ and $\tau=3 \leftrightarrow \tau=4$ can be combined. Because the value of quantum number $k$ must change $(\Delta k=1)$, the torsional-rotational spectrum will form. If the value of quantum number $J$ also changes under the aforementioned conditions, then the intensity will not accumulate because the transition energy will depend on both the torsional quantum numbers and quantum numbers $J^{\prime \prime}$ and $k^{\prime \prime}$. Transitions of this type will not be examined further because, as shown previously [6], the corresponding absorption bands for the IR spectrum will be weak compared with those due to transitions where intensity accumulates. Therefore, considering that $\Delta J=0$, the expression for the Raman line intensity becomes:

$$
I=G N_{n^{\prime \prime} \tau^{\prime \prime}}\left\langle n^{\prime \prime}, \tau^{\prime \prime}\left|\alpha_{0 m}^{(2, \pm 1)}\right| n^{\prime}, \tau^{\prime}\right\rangle^{2} \sum_{J^{\prime \prime}} N_{J^{\prime \prime} k^{\prime \prime}}\left(2 J^{\prime \prime}+1\right)^{2}\left(\begin{array}{ccc}
J^{\prime \prime} & 2 & J^{\prime \prime} \\
k^{\prime \prime} & \pm 1 & -\left(k^{\prime \prime} \pm 1\right)
\end{array}\right)^{2}
$$

Finally, let us examine element $\alpha_{0 m}^{(2, \pm 2)}$. It also is transformed according to $A_{g s}$. Therefore, $\Delta \tau=0$. Furthermore, $\Delta k=$ \pm 2 and $\Delta J=-2,-1,0,1,2$. If the torsional state does not change, the purely rotational Raman spectrum of $\mathrm{H} 2 \mathrm{O} 2$ will form. Accumulation of intensity over rotational states will be observed only for $\Delta J=0$. Then

$$
I=G \sum_{n^{\prime \prime}, \tau^{\prime \prime}} N_{n^{\prime \prime} \tau^{\prime \prime}}\left\langle n^{\prime \prime}, \tau^{\prime \prime}\left|\alpha_{0 m}^{(2, \pm 2)}\right| n^{\prime \prime}, \tau^{\prime \prime}\right\rangle^{2} \sum_{J^{\prime \prime}} N_{J^{\prime \prime} k^{\prime \prime}}\left(2 J^{\prime \prime}+1\right)^{2}\left(\begin{array}{ccc}
J^{\prime \prime} & 2 & J^{\prime \prime} \\
k^{\prime \prime} & \pm 2 & -\left(k^{\prime \prime} \pm 2\right)
\end{array}\right)^{2}
$$

The torsional-rotational spectrum will form for a change of torsional state. If quantum numbers $J$ and $k$ change simultaneously, then a transition without intensity accumulation that is excluded from examination occurs. Therefore, we will henceforth assume that $\Delta J=0$ and that the expression for the Raman line intensity for $\Delta k= \pm 2$ is:

$$
I=G N_{n^{\prime \prime} \tau^{\prime \prime}}\left\langle n^{\prime \prime}, \tau^{\prime \prime}\left|\alpha_{0 m}^{(2, \pm 2)}\right| n^{\prime}, \tau^{\prime \prime}\right\rangle^{2} \sum_{J^{\prime \prime}} N_{J^{\prime \prime} k^{\prime \prime}}\left(2 J^{\prime \prime}+1\right)^{2}\left(\begin{array}{ccc}
J^{\prime \prime} & 2 & J^{\prime \prime} \\
k^{\prime \prime} & \pm 2 & -\left(k^{\prime \prime} \pm 2\right)
\end{array}\right)^{2}
$$

Thus, the purely torsional spectrum forms for elements $\alpha_{0 m}^{(0,0)}$ and $\alpha_{0 m}^{(2,0)}$; rotational, for $\alpha_{0 m}^{(2,0)}$ and $\alpha_{0 m}^{(2, \pm 2)}$; torsional-rotational, for $\alpha_{0 m}^{(2,0)}, \alpha_{0 m}^{(2, \pm 1)}$, and $\alpha_{0 m}^{(2, \pm 2)}$. The Raman line intensity will be determined mainly by the values of the corresponding torsion integrals.

The functional dependence of the polarizability tensor components in the irreducible spherical tensor form on the internal rotation angle $\gamma$ must be found in order to calculate the integrals over the torsional functions. The explicit form of such dependences was obtained based on quantum-chemical calculations using the GAMESS packet $[7,8]$ in the HF/6-311G approximation.

Discussion. Figure 1 shows results of $a b$ initio calculations of the polarizability tensor elements as functions of internal rotation angle. Calculations were performed for torsion angle $\gamma$ in the range $0-180^{\circ}$ in steps of $10^{\circ}$ with fixed dihedral angle and molecular geometry optimized over all other parameters.

It should be noted that the direction of the cartesian CS axes was dependent on $\gamma$ during the calculations using the GAMESS packet. In addition, the intramolecular CS remained fixed relative to the molecule. The CS $x y z$ in which $\gamma=0^{\circ}$ the $x$ axis was parallel to the $\mathrm{OO}$ bond, the $y$ axis lay in the $\mathrm{HOOH}$ plane, and the $z$ axis was perpendicular to this plane was chosen initially. All values of the polarizability tensor components in the range $0^{\circ} \leq \gamma \leq 180^{\circ}$ were calculated in this CS. Components $\alpha_{j l}$ in the range $180^{\circ}<\gamma \leq 360^{\circ}$ were determined using the obvious condition:

$$
\alpha_{j l}(\gamma)=\alpha_{j l}(2 \pi-\gamma)
$$

On the other hand, it is understood that internal rotation in the molecule is complete only upon reaching $\gamma=720^{\circ}$. Therefore, the fixed molecular CS $x^{\prime} y^{\prime} z^{\prime}$, which was related in the examined interval to CS $x y z$ by the condition below, was used to calculate the polarizability tensor components in the range $360^{\circ}<\gamma \leq 720^{\circ}$ : 

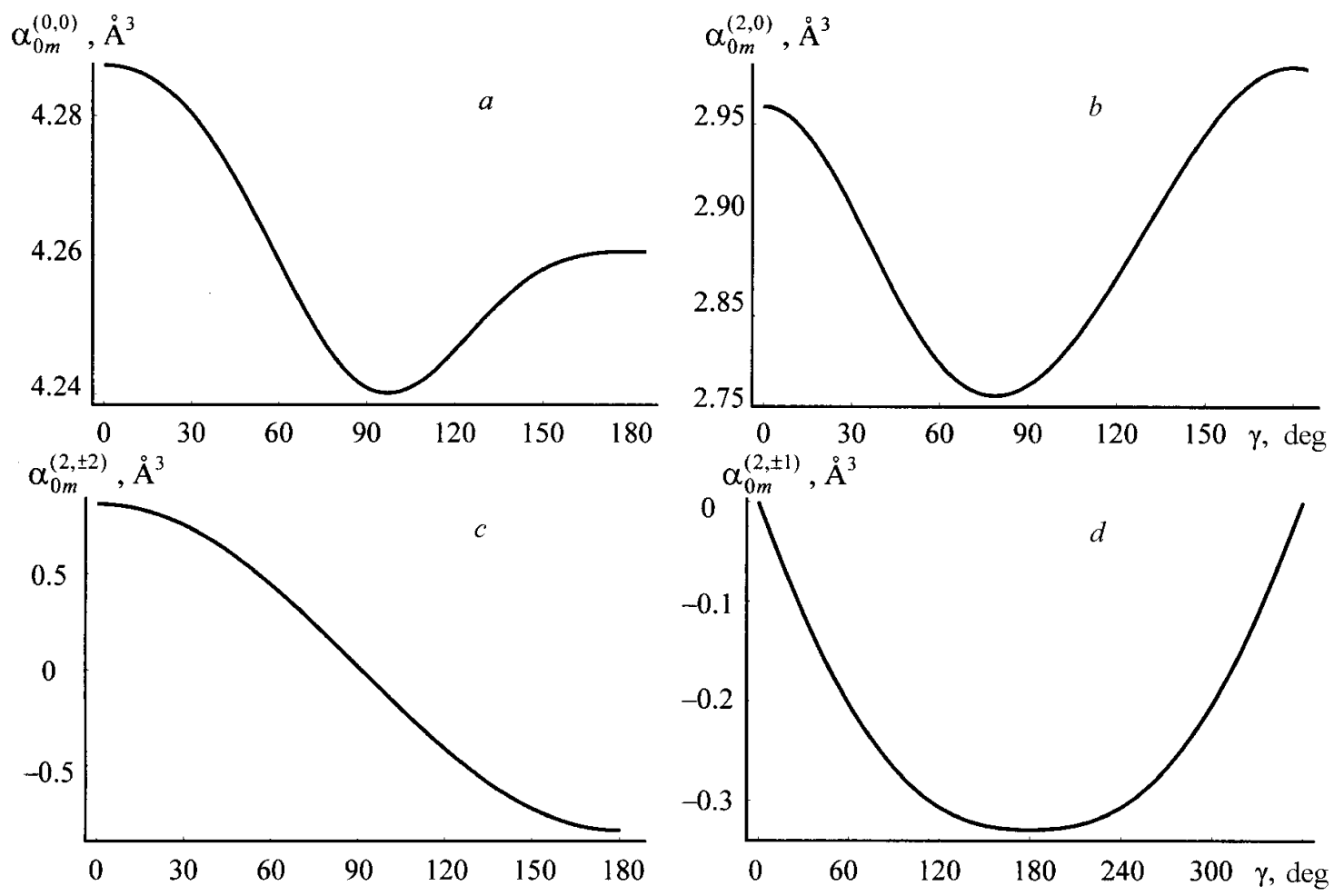

Fig. 1. Components $\alpha_{0 m}^{(0,0)}$ (a), $\alpha_{0 m}^{(2,0)}$ (b), $\alpha_{0 m}^{(2, \pm 2)}$ (c), and $\alpha_{0 m}^{(2, \pm 1)}$ (d) of $\mathrm{H}_{2} \mathrm{O}_{2}$ polarizability tensor as functions of internal rotation angle from ab initio calculations in the HF-6-311G approximation.

$$
\left(\begin{array}{l}
x^{\prime} \\
y^{\prime} \\
z^{\prime}
\end{array}\right)=\left(\begin{array}{rrr}
1 & 0 & 0 \\
0 & -1 & 0 \\
0 & 0 & -1
\end{array}\right)\left(\begin{array}{l}
x \\
y \\
z
\end{array}\right) .
$$

The polarizability tensor diagonal components $\alpha_{j j}$ for this transformation did not change $\left[\alpha_{j^{\prime} j^{\prime}}=( \pm 1)^{2} \alpha_{j j}\right]$ whereas component $\alpha_{x z}$ changes sign $\left[\alpha_{x^{\prime} z^{\prime}}=(+1)(-1) \alpha_{x z}\right]$. Thanks to this, components of the type $\alpha_{j j}$ became $2 \pi$-periodic whereas component $\alpha_{x z}$ remained $4 \pi$-periodic. Thus, according to Eqs. (5), (8), and (9), elements $\alpha_{0 m}^{(0,0)}, \alpha_{0 m}^{(2,0)}$, $\alpha_{0 m}^{(2, \pm 2)}$ and were even functions of $\gamma$ with a $2 \pi$ period; $\alpha_{0 m}^{(2, \pm 1)}$, an uneven function with a $4 \pi$ period.

Table 1 presents several squares of matrix elements of the polarizability tensor components for lower torsional states. Energies of torsional and rotational states that were calculated beforehand were used to calculate frequencies of torsional and rotational transitions [6]. Values of the rotational constants $\left(A=9.960 \mathrm{~cm}^{-1}, B=C=0.901 \mathrm{~cm}^{-1}\right)$ were obtained using experimental geometric parameters [4].

Raman line intensities of the torsional spectrum were calculated for $n=\overline{0,5}$ and $\tau=1,2,3,4$. The calculation of the torsional spectrum showed that line intensities due to element $\alpha_{0 m}^{(0,0)}$ of Eq. (12) were three orders of magnitude less than the corresponding lines for element $\alpha_{0 m}^{(2,0)}$ of Eq. (13). Figure 2 shows the calculated Raman torsional spectrum.

The Raman rotational spectrum of $\mathrm{H}_{2} \mathrm{O}_{2}$ was formed from three line progressions with different periods and intensities. The first progression was due to element $\alpha_{0 m}^{(2,0)}$ of Eq. (14) and selection rule $\Delta J=1$. It was situated in the range $3-70 \mathrm{~cm}^{-1}$ with period $2 B\left(1.802 \mathrm{~cm}^{-1}\right)$ and had the greatest line intensity at $19.823 \mathrm{~cm}^{-1}(J=10)$. The second line progression was also due to element $\alpha_{0 m}^{(2,0)}$ but for selection rule $\Delta J=2$. This progression covered the range $5-170 \mathrm{~cm}^{-1}$ with period $4 B\left(3.604 \mathrm{~cm}^{-1}\right)$ with greatest line intensity at $59.470 \mathrm{~cm}^{-1}(J=15)$. The third pro- 
TABLE 1. Squares of Polarizability Tensor Matrix Elements Components for Low Torsional States

\begin{tabular}{|c|c|c|c|c|}
\hline$n^{\prime \prime}, \tau^{\prime \prime} \rightarrow n^{\prime}, \tau^{\prime}$ & $\left\langle n^{\prime \prime}, \tau^{\prime \prime}\left|\alpha_{0 m}^{(0,0)}\right| n^{\prime}, \tau^{\prime}\right\rangle^{2}$ & $\left\langle n^{\prime \prime}, \tau^{\prime \prime}\left|\alpha_{0 m}^{(2,0)}\right| n^{\prime}, \tau^{\prime}\right\rangle^{2}$ & $\left\langle n^{\prime \prime}, \tau^{\prime \prime}\left|\alpha_{0 m}^{(2, \pm 1)}\right| n^{\prime}, \tau^{\prime}\right\rangle^{2}$ & $\left\langle n^{\prime \prime}, \tau^{\prime \prime}\left|\alpha_{0 m}^{(2, \pm 2)}\right| n^{\prime}, \tau^{\prime}\right\rangle^{2}$ \\
\hline $\begin{array}{l}0,1 \rightarrow 0,1 \\
0,2 \rightarrow 0,2\end{array}$ & 6.015 & 1.378 & 0 & $3.697 \cdot 10^{-2}$ \\
\hline $0,1 \rightarrow 0,2$ & 0 & 0 & $9.693 \cdot 10^{-2}$ & 0 \\
\hline $\begin{array}{l}0,3 \rightarrow 0,3 \\
0,4 \rightarrow 0,4\end{array}$ & 6.011 & 1.369 & 0 & $2.860 \cdot 10^{-2}$ \\
\hline $0,3 \rightarrow 0,4$ & 0 & 0 & $8.865 \cdot 10^{-2}$ & 0 \\
\hline $\begin{array}{l}0,1 \rightarrow 1,1 \\
0,2 \rightarrow 1,2\end{array}$ & $1.151 \cdot 10^{-5}$ & $3.382 \cdot 10^{-4}$ & 0 & $1.401 \cdot 10^{-2}$ \\
\hline $0,1 \rightarrow 1,2$ & 0 & 0 & $3.167 \cdot 10^{-4}$ & 0 \\
\hline $\begin{array}{l}0,3 \rightarrow 1,3 \\
0,4 \rightarrow 1,4\end{array}$ & $7.875 \cdot 10^{-6}$ & $2.580 \cdot 10^{-4}$ & 0 & $1.401 \cdot 10^{-2}$ \\
\hline $0,3 \rightarrow 1,4$ & 0 & 0 & $3.895 \cdot 10^{-4}$ & 0 \\
\hline $\begin{array}{l}0,1 \rightarrow 2,1 \\
0,2 \rightarrow 2,2\end{array}$ & $3.991 \cdot 10^{-9}$ & $8.446 \cdot 10^{-6}$ & 0 & $2.449 \cdot 10^{-3}$ \\
\hline $0,1 \rightarrow 2,2$ & 0 & 0 & $1.210 \cdot 10^{-4}$ & 0 \\
\hline $\begin{array}{l}0,3 \rightarrow 2,3 \\
0,4 \rightarrow 2,4\end{array}$ & $1.805 \cdot 10^{-6}$ & $4.376 \cdot 10^{-6}$ & 0 & $3.550 \cdot 10^{-4}$ \\
\hline $0,3 \rightarrow 2,4$ & 0 & 0 & $4.489 \cdot 10^{-5}$ & 0 \\
\hline $\begin{array}{l}1,1 \rightarrow 1,1 \\
1,2 \rightarrow 1,2\end{array}$ & 6.031 & 1.417 & 0 & $6.973 \cdot 10^{-2}$ \\
\hline $1,1 \rightarrow 1,2$ & 0 & 0 & $9.477 \cdot 10^{-2}$ & 0 \\
\hline $\begin{array}{l}1,3 \rightarrow 1,3 \\
1,4 \rightarrow 1,4\end{array}$ & 6.022 & 1.383 & 0 & $2.904 \cdot 10^{-2}$ \\
\hline $1,3 \rightarrow 1,4$ & 0 & 0 & $8.613 \cdot 10^{-2}$ & 0 \\
\hline $\begin{array}{l}1,1 \rightarrow 2,1 \\
1,2 \rightarrow 2,2\end{array}$ & $7.516 \cdot 10^{-6}$ & $3.990 \cdot 10^{-4}$ & 0 & $1.936 \cdot 10^{-2}$ \\
\hline $1,1 \rightarrow 2,2$ & 0 & 0 & $4.998 \cdot 10^{-4}$ & 0 \\
\hline $\begin{array}{l}1,3 \rightarrow 2,3 \\
1,4 \rightarrow 2,4\end{array}$ & $3.771 \cdot 10^{-6}$ & $3.269 \cdot 10^{-4}$ & 0 & $2.329 \cdot 10^{-2}$ \\
\hline $1,3 \rightarrow 2,4$ & 0 & 0 & $7.552 \cdot 10^{-4}$ & 0 \\
\hline
\end{tabular}

gression had period $4(A-B)\left(36.236 \mathrm{~cm}^{-1}\right)$ and was due to element $\alpha_{0 m}^{(2, \pm 2)}$ of Eq. (15). The line intensities of this progression decreased smoothly starting at a frequency equal to the progression period.

Line intensities of the Raman rotational spectrum were calculated for $n=\overline{0,9} ; \tau=1,2,3$, 4; and $J=$ $\overline{0,60}$. Figure 3 shows the resulting Raman rotational spectrum. It is important to note that the intensities of spectral lines in the rotational spectrum were three orders of magnitude greater than those in the torsional spectrum, which was due mainly to differences of the diagonal and off-diagonal matrix elements (Table 1).

The contributions of elements $\alpha_{0 m}^{(2,0)}$ and $\alpha_{0 m}^{(2, \pm 1)}$ to the formation of the Raman torsional-rotational spectrum of $\mathrm{H}_{2} \mathrm{O}_{2}$ were negligibly small compared with that of $\alpha_{0 m}^{(2, \pm 2)}$. Therefore, the contribution of only this element of the polarizability tensor was examined further. Line intensities of the torsional-rotational Raman spectrum were calculated for $n=\overline{0,3} ; \tau=1,2,3,4$; and $k=\overline{0,11}$. 


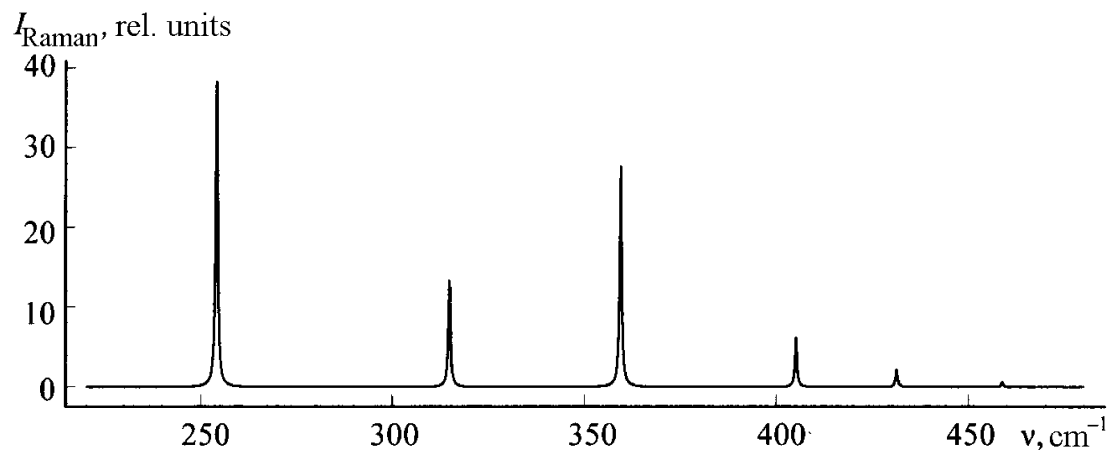

Fig. 2. Torsional Raman spectrum of $\mathrm{H}_{2} \mathrm{O}_{2}$.

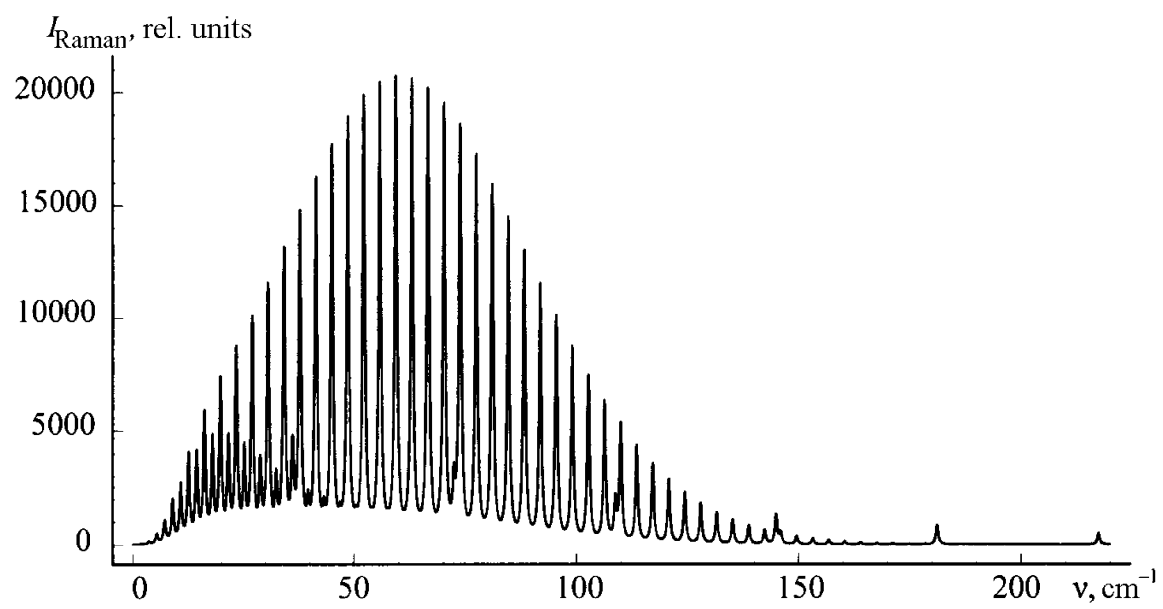

Fig. 3. Rotational Raman spectrum of $\mathrm{H}_{2} \mathrm{O}_{2}$.

The transition between a system of torsional-rotational states belonging to a given $n^{\prime \prime}$ and a certain other $n^{\prime}$ formed two band progressions where both progressions had the same period of $4(A-B)=36.236 \mathrm{~cm}^{-1}$. Let us examine $n^{\prime \prime}=0$ and $n^{\prime}=1$ because transitions between adjacent $n$ values were the strongest.

Transitions between states with $\tau=1$ and 2 formed the first progression, the center of which was located at $290.384 \mathrm{~cm}^{-1}$ and corresponded to transitions with $\Delta J=0, \tau^{\prime \prime}=\tau^{\prime}=1$, and $k^{\prime \prime}=0, k^{\prime}=2$. Lines with higher frequencies were formed by transitions with $\Delta k=+2$; with lower frequencies, transitions with $\Delta k=-2$ (Fig. 4a). An analogous progression for transitions $n^{\prime \prime}=0 \rightarrow n^{\prime}=2$ was located in the range $300-900 \mathrm{~cm}^{-1}$. Transitions between states with $\tau=3$ and 4 formed a second progression, the center of which was located at $395.560 \mathrm{~cm}^{-1}$ and corresponded to transitions with $\Delta J=0, \tau^{\prime \prime}=\tau^{\prime}=4$, and $k^{\prime \prime}=0, k^{\prime}=2$. Lines with higher frequencies were formed by transitions with $\Delta k=+2$; with lower ones, with $\Delta k=-2$ (Fig. 4b). An analogous progression for transitions $n^{\prime \prime}=0$ $\rightarrow n^{\prime}=2$ was located in the range $600-1000 \mathrm{~cm}^{-1}$. The frequency difference of the progression centers was determined by the following condition (the first index is the $n$ value; the second, $\tau$ ):

$$
\left(E_{1,4}-E_{0,4}\right)-\left(E_{1,1}-E_{0,1}\right)=105.176 \mathrm{~cm}^{-1} .
$$

Lines of the two progressions were by chance in close proximity $\left(3.531 \mathrm{~cm}^{-1}\right)$.

Progressions for transitions $n^{\prime \prime}=1 \rightarrow n^{\prime}=2$ (center of the first progression located at $351.064 \mathrm{~cm}^{-1}$; of the second, $441.280 \mathrm{~cm}^{-1}$, difference $91.216 \mathrm{~cm}^{-1}$ ) were formed analogously. The center of the first progression for transitions $n^{\prime \prime}=2 \rightarrow n^{\prime}=3$ was situated at $467.498 \mathrm{~cm}^{-1}$; of the second, $495.047 \mathrm{~cm}^{-1}$, difference $27.549 \mathrm{~cm}^{-1}$. The 

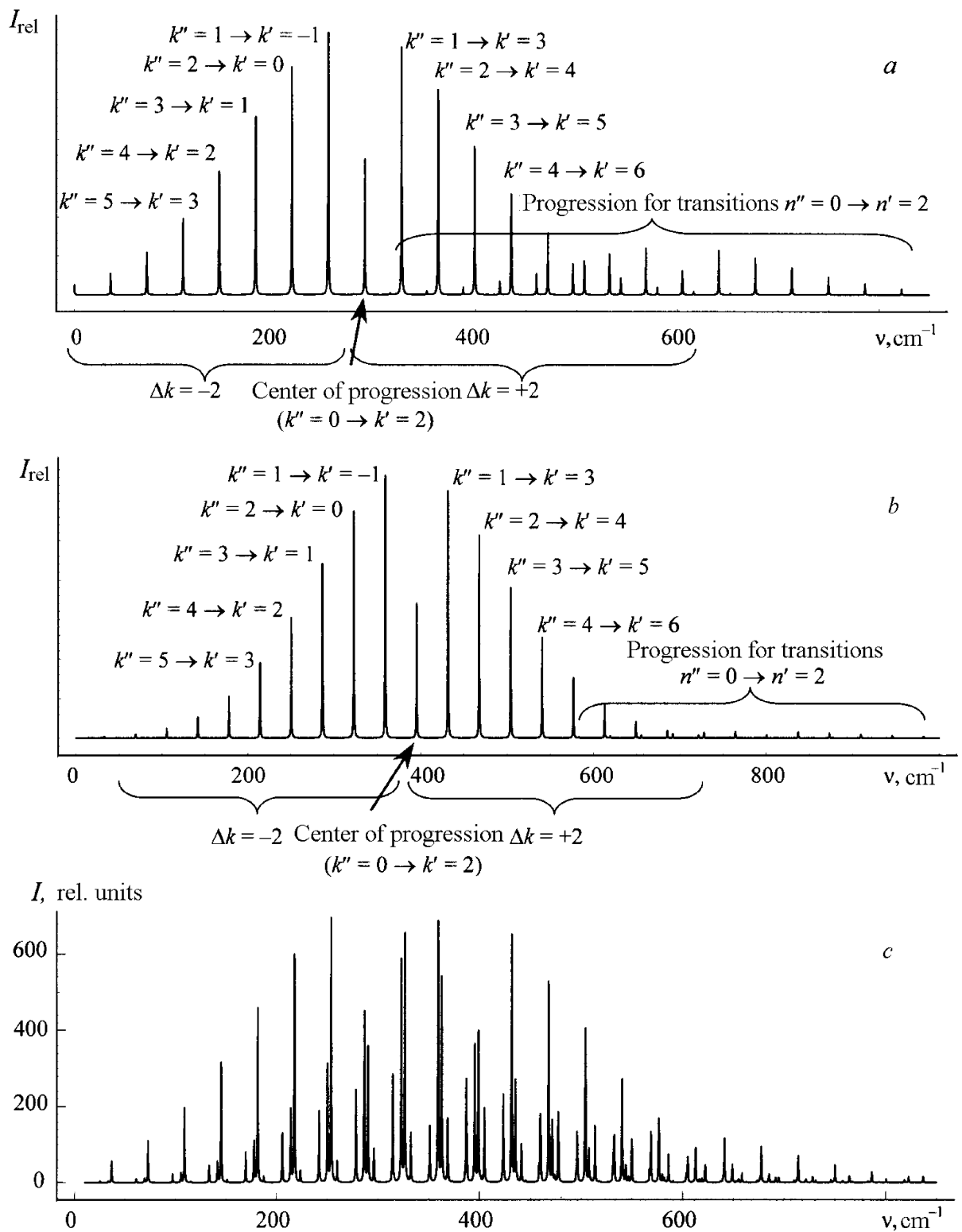

$I$, rel. units

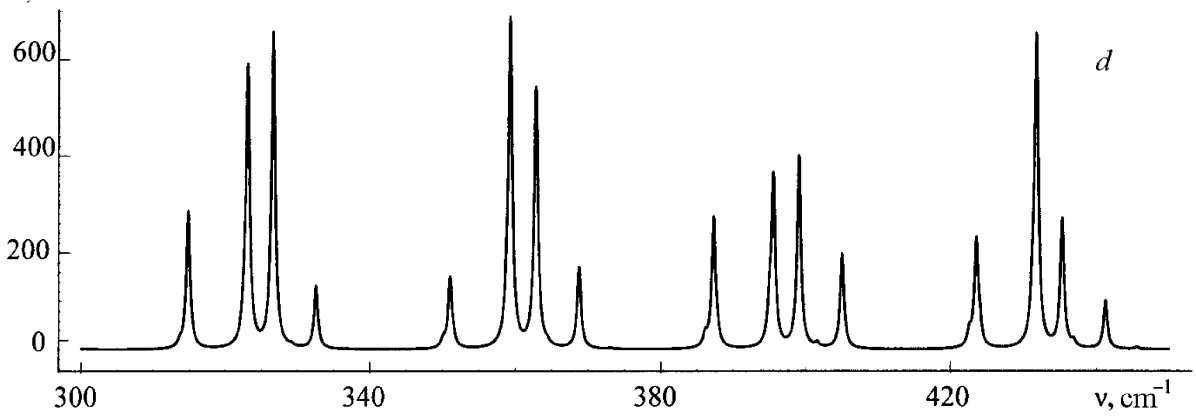

Fig. 4. Torsional-rotational Raman spectrum of $\mathrm{H}_{2} \mathrm{O}_{2}$ : formation of line progression for transition $n^{\prime \prime}=0 \rightarrow n^{\prime}=1, \tau=1$ and 2 (a); $\tau=3$ and 4 (b); spectrum including transitions up to $n=3$ (c); fine structure of spectral lines (d). 
center of the first progression for transitions $n^{\prime \prime}=3 \rightarrow n^{\prime}=4$ was located at $509.377 \mathrm{~cm}^{-1}$; of the second, 518.047 $\mathrm{cm}^{-1}$; difference $8.670 \mathrm{~cm}^{-1}$. Transitions between states with higher $\mathrm{n}$ values contributed little to the torsional-rotational Raman spectrum. Figure $4 \mathrm{c}$ shows the resulting spectrum. Figure $4 \mathrm{~d}$ shows a fragment of the torsional-rotational spectrum that demonstrates the fine structure. Line intensities of the torsional-rotational spectrum were $\approx 30$ times less than those of the rotational spectrum.

Obviously the resulting Raman spectrum of $\mathrm{H}_{2} \mathrm{O}_{2}$ in the long-wavelength region is the sum of the three spectra shown in Figs. 2, 3, and 4c. Lines of the rotational spectrum dominate in the region $<200 \mathrm{~cm}^{-1}$; of the torsionalrotational spectrum, at $200-600 \mathrm{~cm}^{-1}$. Because lines of the purely torsional spectrum also fall in this region whereas their intensities are an order of magnitude less, it is improbable that the torsional spectrum of $\mathrm{H}_{2} \mathrm{O}_{2}$ is observed.

Conclusion. It was found that the structure and transformational properties of the polarizability tensor components of $\mathrm{H} 2 \mathrm{O} 2$ in the EMS group $G_{4}(\mathrm{EM})$ allow the formation of purely rotational and torsional in addition to torsional-rotational Raman spectra. This is an important difference from the situation with the IR spectra.

General expressions for calculating Raman line intensities of a symmetric-top non-rigid molecule are obtained and used to model torsional, rotational, and torsional-rotational Raman spectra of $\mathrm{H}_{2} \mathrm{O}_{2}$. The relative intensities of the various types of Raman spectra are determined mainly by matrix elements of the polarizability tensor components over torsional functions whereas Raman line intensities in each of the spectra are due mainly to summations of transitions with identical energy over rotational or torsional quantum numbers.

\section{REFERENCES}

1. R. H. Hunt, R. A. Leacock, C. W. Peters, and K. T. Hecht, J. Chem. Phys., 42, 1931-1942 (1965).

2. P. Helminger, W. C. Bowman, and F. C. DeLucia, J. Mol. Spectrosc., 85, 120-130 (1981).

3. J. J. Hillman, D. E. Jennings, W. B. Olson, and A. Goldman, J. Mol. Spectrosc., 117, 46-59 (1986).

4. J.-M. Flaud, C. Camy-Peyret, J. W. C. Johns, and B. Carli, J. Chem. Phys., 91, 1504-1510 (1989).

5. C. Camy-Peyret, J.-M. Flaud, J. W. C. Johns, and M. Noel, J. Mol. Spectrosc., 155, 84-104 (1992).

6. A. V. Shashok, M. B. Shundalau, and G. A. Pitsevich, Zh. Prikl. Spektrosk., 75, No. 2, 153-158 (2008).

7. http://www.msg.ameslab.gov/GAMESS/GAMESS.html.

8. M. W. Schmidt, K. K. Baldridge, J. A. Boatz, S. T. Elbert, M. S. Gordon, J. H. Jensen, S. Koseki, N. Matsunaga, K. A. Nguyen, S. J. Su, T. L. Windus, M. Dupuis, and J. A. Montgomery, J. Comput. Chem., 14, 13471363 (1993).

9. M. A. El'yashevich, Atomic and Molecular Spectroscopy [in Russian], URSS, Moscow (2001).

10. P. R. Bunker and P. Jensen, Molecular Symmetry and Spectroscopy, NRC Research Press, Ottawa (1998).

11. R. N. Zare, Angular Momentum, Wiley, New York (1988). 